\title{
205 Late enhancement in contrast-enhanced cardiac MRI facilitates the characterization of different etiologies in non-ischemic myocardial disease
}

\author{
Peter Hunold*1, Horst Franzen ${ }^{1}$, Oliver Bruder ${ }^{2}$, Thomas Schlosser ${ }^{1}$, \\ Kai Nassenstein ${ }^{1}$, Markus Jochims² ${ }^{2}$ Georg V Sabin ${ }^{2}$ and Jörg Barkhausen ${ }^{1}$
}

Address: ${ }^{1}$ University Hospital Essen, Essen, Germany and ${ }^{2}$ Elisabeth Hospital, Essen, Germany

* Corresponding author

from I th Annual SCMR Scientific Sessions

Los Angeles, CA, USA. I-3 February 2008

Published: 22 October 2008

Journal of Cardiovascular Magnetic Resonance 2008, I0(Suppl I):A66 doi:10.1 I86/1532-429X-I0-SI-A66

This abstract is available from: http://jcmr-online.com/content/I0/SI/A66

(c) 2008 Hunold et al; licensee BioMed Central Ltd.

\section{Introduction}

Late gadolinium enhancement (LGE) in contrastenhanced MRI (CE-MRI) has been proven to reliably identify and quantify scar after myocardial infarction in coronary artery disease (CAD). However, LGE is not specific for ischemic damage since different fibrotic and inflammatory diseases with enlarged interstitial volume are accompanied by LGE. During recent years, many studies have been performed showing the clinical usefulness of the additional information given by LGE imaging. Different myocardial diseases seem to present with specific patterns of LGE enabling to differentiate different etiologies.

\section{Purpose}

Aim of this study was to review cases of LGE not caused by myocardial infarction in a large collective of consecutive cardiac MRI examinations and to characterize specific patterns of LE in different groups of non-ischemic heart disease.

\section{Methods}

Within 50 months, a total of 5676 contrast-enhanced cardiac MRI studies were performed on different $1.5 \mathrm{~T}$ scanners (Magnetom Sonata (2) and Avanto (1), Siemens, Germany) in two affiliated institutions for different clinical indications. The uniformly utilized MRI protocol consisted of a functional study in standard long axis and contiguous short axis orientations of the entire left ventricle using a segmented TrueFISP sequence. Data sets for
LGE detection were acquired 8-15 min after administration of $0.2 \mathrm{mmol} / \mathrm{kg} \mathrm{BW}$ of Gd-based extracellular contrast agents in the same orientations using a segmented inversion-recovery TurboFLASH sequence (TR, $8 \mathrm{~ms}$; TE, 4 ms; TI, 200-260 ms; slice thickness, $8 \mathrm{~mm}$ ). All cases of non-ischemic LGE were retrospectively collected and reviewed. The different patterns of LE were related to the underlying pathology as stated by means of clinical and other diagnostic imaging features.

\section{Results}

A total of 1905 (34\%) patients presented with LGE. All cases of confirmed ischemic infarction $(1644,28 \%)$ presented with typical subendocardial or transmural, strongly hyperintense LGE with sharp demarcation. 261 (4.6\%) patients yielded different patterns of non-ischemic LGE:

A) Mid-myocardial/intramural LGE, 130/261 (50\%): e.g. acute or chronic myocarditis hypertrophic cardiomyopathy, dilative cardiomyopathy, Fabry's disease etc.

B) Subepicardial LGE, 48/261 (18\%): acute or chronic myocarditis, etc.

C) Transmural LGE, 18/261 (7\%): Chronic myocarditishypertrophic CMP, Fabry's disease etc. 
D) Other kind of LGE, 58/261 (22\%): Fibrosis in aortic valve stenosis, hypertrophic $\mathrm{CMP}$, microembolization after PCI, vasculitis etc.

Within the different LGE pattern groups, remarkable differences in pattern, intensity, and localization existed to define the underlying pathology. In general, non-infarction LGE could well be distinguished from ischemic damage because of characteristic LGE patterns.

\section{Conclusion}

After establishing the Late Gd enhancement technique in myocardial viability imaging, multiple applications have recently been arising in non-ischemic disease. Many nonischemic myocardial diseases are accompanied by LGE because of higher contrast material concentration in fibrosis or edema. Different groups of myocardial diseases seem to present with specific LGE patterns and localizations allowing for both, differentiation from ischemic myocardial damage and differential diagnosis of the underlying pathology. It is now time to define and catalog these specific appearances.
Publish with Biomed Central and every scientist can read your work free of charge

"BioMed Central will be the most significant development for disseminating the results of biomedical research in our lifetime. " Sir Paul Nurse, Cancer Research UK

Your research papers will be:

- available free of charge to the entire biomedical community

- peer reviewed and published immediately upon acceptance

- cited in PubMed and archived on PubMed Central

- yours - you keep the copyright

Submit your manuscript here:

http://www.biomedcentral.com/info/publishing_adv.asp 